\title{
Influence of $\mathrm{O}$ and $\mathrm{C}$ co-implantation on the lattice site of $\mathrm{Er}$ in $\mathrm{GaN}$
}

\author{
B. De Vries, ${ }^{a}{ }^{2}$ V. Matias, and A. Vantomme \\ Instituut voor Kern- en Stralingsfysica, K. U. Leuven, Celestijnenlaan 200 D, 3001 Leuven, Belgium \\ U. Wahl, E. M. C. Rita, and E. Alves \\ Instituto Tecnológico e Nuclear, Estrada Nacional 10, 2686-953 Sacavém, Portugal \\ A. M. L. Lopes \\ Departamento de Física, Universidade de Aveiro, Campus de Santiago, 3810-193 Aveiro, Portugal \\ J. G. Correia and The ISOLDE Collaboration \\ CERN-EP, 1211 Geneva 23, Switzerland
}

(Received 26 February 2004; accepted 29 March 2004; published online 7 May 2004)

\begin{abstract}
The lattice location of low-dose implanted $\mathrm{Er}$ in $\mathrm{GaN}, \mathrm{GaN}: \mathrm{O}$, and $\mathrm{GaN}: \mathrm{C}$ was investigated using the emission channeling technique. The conversion electrons emitted by the probe isotope ${ }^{167 m} \mathrm{Er}$ give direct evidence that the majority $(\approx 90 \%)$ of Er atoms are located on substitutional Ga sites for all samples. Annealing up to $900^{\circ} \mathrm{C}$ does not change these fractions, although it reduces the $\mathrm{Er}$ root-mean-square (rms) displacements. The only visible effect of oxygen or carbon doping is a small increase in the rms displacements with respect to the undoped sample. (C) 2004 American Institute of Physics. [DOI: 10.1063/1.1756196]
\end{abstract}

Due to its optoelectronic properties, rare earth implanted $\mathrm{GaN}$ is an intensively studied system. Besides its luminescence in the infrared, the green luminescence from Er, in addition to red $(\mathrm{Eu})$ and blue $(\mathrm{Tm})$ light emission, also opens perspectives for manufacturing rare earth implanted $\mathrm{GaN}$ flat panel displays. ${ }^{1}$ It is known that in the case of $\mathrm{Er}$ in $\mathrm{Si}$, co-doping or co-implantation with oxygen drastically increases the luminescence intensity ${ }^{2}$ and changes the lattice site of Er. ${ }^{3}$ With respect to the effect of $\mathrm{O}$ on Er luminescence in GaN, varying results are found in the literature. ${ }^{4}$ While some articles ${ }^{5-7}$ state a beneficial influence on the luminescence, others report no obvious benefit ${ }^{8}$ or even a negative impact ${ }^{9-11}$ on Er emission intensity. It has been pointed out ${ }^{4,9}$ that the annealing temperature is possibly crucial, with $\mathrm{O}$ co-doping being beneficial only for $\mathrm{GaN}$ samples that are not annealed above $900{ }^{\circ} \mathrm{C}$. Finally, codoping with $\mathrm{C}$ has also been claimed to increase the luminescence. ${ }^{12}$

Several publications have already addressed the lattice location of $\mathrm{Er}$ in $\mathrm{GaN}$ and GaN:O. Rutherford backscattering/channeling $(\mathrm{RBS} / \mathrm{C})$ experiments performed for higher doses $\left(\approx 10^{15} \mathrm{~cm}^{-2}\right)$ of implanted $\mathrm{Er}$ found $50 \%-70 \%$ on substitutional $\mathrm{Ga}\left(\mathrm{S}_{\mathrm{Ga}}\right)$ sites. ${ }^{6,10}$ It was also concluded that the addition of oxygen stabilizes $\mathrm{Er}$ on $\mathrm{Ga}$ sites. Another RBS/C study ${ }^{11}$ shows $32 \%$ of Er on $\mathrm{S}_{\mathrm{Ga}}$ sites while a fraction of $13 \%$ is displaced along the [0001] direction. A ${ }^{167 m}$ Er emission channeling experiment ${ }^{13}$ found 90 $\pm 10 \%$ of Er along the [0001] direction with a root mean square (rms) displacement of $0.25 \AA$, irrespective of a virgin sample or previous $\mathrm{O}$ co-implantation.

Our lattice location study was performed using emission channeling. This technique is based on the fact that charged particles emitted during the decay of radioactive isotopes are guided by the potential of rows and planes of atoms while

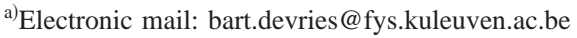

traveling through a crystalline solid. Depending on the lattice sites occupied by the emitter isotopes, characteristic channeling or blocking emission patterns emerge around the major crystal axes and planes. By measuring these angle-dependent emission yields and fitting them to theoretically calculated patterns, the lattice site(s) occupied by the emitting isotopes can be deduced. By using position sensitive detectors and by measuring along four independent crystal axes we achieve a significantly higher precision than the previous studies. An elaborate discussion of the technique, the experimental setup and analysis procedures can be found elsewhere. ${ }^{14,15}$

The radioactive probe isotope that was used in this study is ${ }^{167} \mathrm{Tm}$, which decays into the isomeric excited state ${ }^{167 m} \operatorname{Er}\left(t_{1 / 2}=2.27 \mathrm{~s}\right)$. In the subsequent decay to the ground state conversion electrons of 150, 199, and $206 \mathrm{keV}$ are emitted, which give direct evidence of the lattice site(s) occupied by $\mathrm{Er}$ in the host crystal. ${ }^{3}$ Note that, since ${ }^{167} \mathrm{Tm}$ is implanted, any complexes of $\mathrm{O}$ or $\mathrm{C}$ in the pre-implanted samples will initially be formed with Tm. However, we do not consider this a serious limitation of our studies due to the chemical similarity of the rare earth elements and the fact that the nuclear recoil during the electron capture decay of ${ }^{167} \mathrm{Tm}$ to ${ }^{167 m} \mathrm{Er}$ is only $0.7-0.9 \mathrm{eV}$, which is insufficient to reimplant the Er atom.

Implantations of $60 \mathrm{keV}{ }^{167} \mathrm{Tm}$ were performed at the ISOLDE facility of CERN to doses of $2.0 \times 10^{13} \mathrm{~cm}^{-2}$ into three $\mathrm{GaN}$ samples grown by metalorganic chemical vapor deposition (MOCVD): one virgin film and two that were pre-implanted with $11 \mathrm{keV}{ }^{16} \mathrm{O}$ or $8 \mathrm{keV}{ }^{12} \mathrm{C}$, respectively, both to a dose of $5.0 \times 10^{14} \mathrm{~cm}^{-2}$. The $\mathrm{O}$ and $\mathrm{C}$ implantation energies and doses were chosen in such a way that their peak concentrations overlap with the one of ${ }^{167} \mathrm{Tm} 160 \AA$ beneath the GaN surface, resulting in Tm:O and Tm:C concentration ratios of $\sim 1: 14$. Subsequently, the angular electron emission patterns were measured at room temperature along the [0001], [ $\overline{1} 102],[\overline{1} 101]$, and [ $\overline{2} 113]$ axes for the 


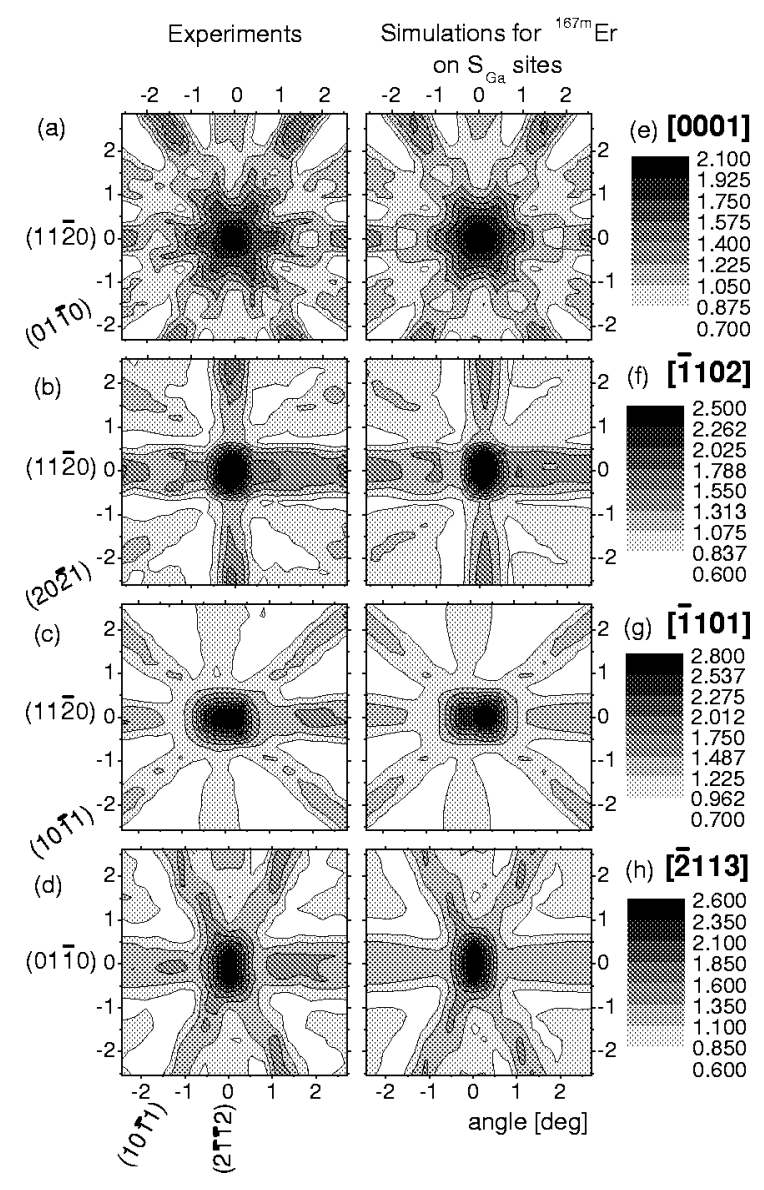

FIG. 1. (a)-(d) Angular-dependent emission yields of conversion electrons emitted from ${ }^{167 m} \mathrm{Er}$ in GaN around the [0001], [11102], [ $\left.\overline{1} 101\right]$, and [ 2113$]$ axes following $800^{\circ} \mathrm{C}$ vacuum annealing; (e)-(h) best fits of theoretical patterns to the experimental yields, corresponding to $\mathrm{S}_{\mathrm{Ga}}$ fractions of $95 \%$, $88 \%, 88 \%$, and $93 \%$ and rms displacements $u_{1}$ perpendicular to the indicated axes of $0.12,0.10,0.10$, and $0.14 \AA$, respectively.

as-implanted state as well as after isochronal $(10 \mathrm{~min})$ vacuum annealing steps up to $900^{\circ} \mathrm{C}$. Note that we started with three identical GaN samples, and that they were measured using the same experimental setup under the same conditions. Additionally, because only low doses of ${ }^{167} \mathrm{Tm}$ are implanted, clustering of probe atoms is avoided. The differences in results between the samples should therefore only depend on the influence of oxygen or carbon on isolated $\mathrm{Er}$ atoms in the GaN crystal.

Figure 1(a) shows the normalized angular emission yield of conversion electrons around the $c$ axis of the virgin sample after $800^{\circ} \mathrm{C}$ annealing. The central axial channeling peak and the intersecting planar channeling effects are direct evidence that a substantial fraction of the emitter atoms are situated along the [0001] atom rows. However, since the [0001] atom rows consist of alternating $\mathrm{Ga}$ and $\mathrm{N}$ atoms, it is impossible to deduce from pattern (a) alone whether the ${ }^{167 m} \mathrm{Er}$ atoms are located on $\mathrm{S}_{\mathrm{Ga}}$ or $\mathrm{S}_{\mathrm{N}}$ sites or on one of the several interstitial sites along these rows. It is therefore essential to also measure along at least one other crystal axis that does not have mixed $\mathrm{Ga}$ and $\mathrm{N}$ rows in order to fully determine the lattice site location. Figures 1(b)-1(d) show additional emission channeling patterns measured around the [11102], [ $\overline{1} 101]$, and [ $\overline{2} 113]$ axes. Since all of these directions consist of pure $\mathrm{Ga}$ and $\mathrm{N}$ atomic rows, they allow the Downloaded 05 Aug 2004 to 141.211.175.139. Redistribution subje

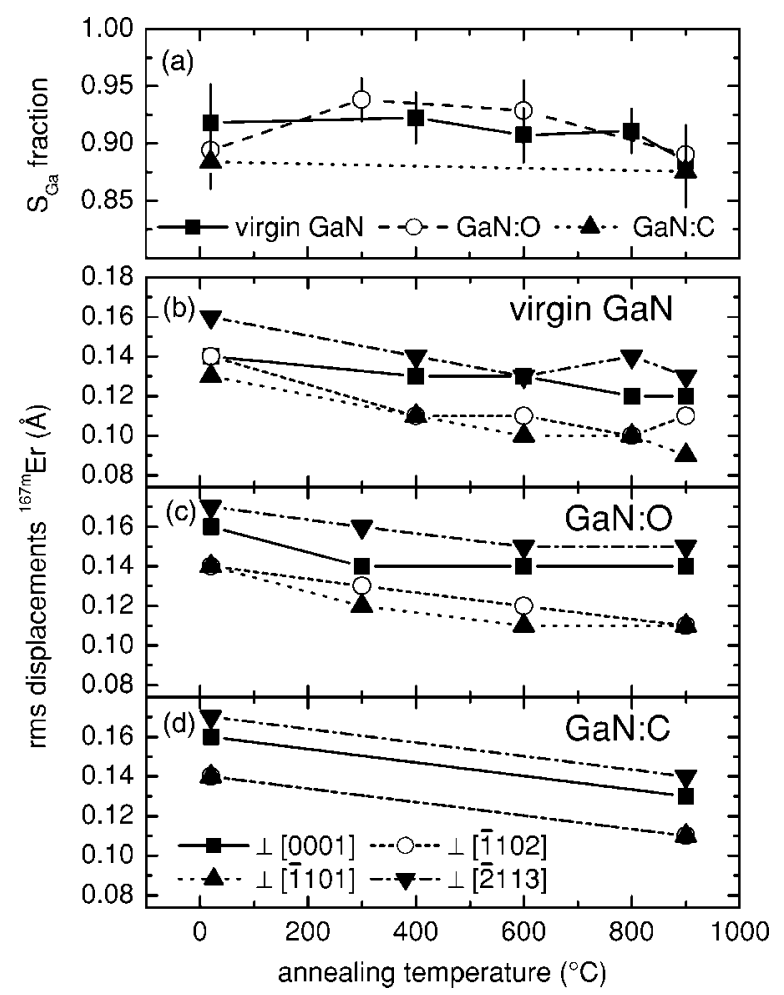

FIG. 2. (a) $\mathrm{S}_{\mathrm{Ga}}$ fractions as a function of annealing temperature. Each data point is actually the mean of the $\mathrm{S}_{\mathrm{Ga}}$ fractions obtained for the four channeling directions. Error bars indicate statistical errors only. The remainder of $\mathrm{Er}$ atoms is located on random or low symmetry sites, although small fractions $(<5 \%)$ of Er on other high symmetry sites would still be in agreement with the analysis; (b)-(d): rms displacements perpendicular to the channeling axes, measured as a function of isochronal annealing temperature. The vertical scale offset corresponds to the vibration amplitude of $\mathrm{Ga}$ in $\mathrm{GaN}$, $u_{1}(\mathrm{Ga})=0.0739 \AA$.

unambiguous determination of the lattice sites of ${ }^{167 m} \mathrm{Er}$. By fitting all patterns to theoretically calculated patterns for emitters on different lattice sites of the GaN crystal, one can deduce quantitative information: the best fits [Figs. 1(e)1(h)] result in $91(3) \%$ of ${ }^{167 m} \mathrm{Er}$ atoms on Ga sites with rms displacements of $0.12,0.10,0.10$, and $0.14 \AA$ perpendicular to the corresponding channeling directions. The remainder is located on random sites or low symmetry sites.

An overview of the substitutional fractions found for all three samples as a function of annealing temperature is given in Fig. 2(a). It is observed that large fractions $(\approx 90 \%)$ of ${ }^{167 m} \mathrm{Er}$ are located on $\mathrm{S}_{\mathrm{Ga}}$ sites already after room temperature implantation for all three samples. Annealing up to $900{ }^{\circ} \mathrm{C}$ influences the substitutional fractions only slightly, with intermediate temperatures giving the highest yields. From these results it is obvious that the presence of $\mathrm{O}$ or $\mathrm{C}$ does not noticeably influence the lattice sites occupied by $\mathrm{Er}$ in $\mathrm{GaN}$, at least not for low doses of $2.0 \times 10^{13} \mathrm{~cm}^{-2}$, in striking contrast to $\mathrm{O}$ in $\mathrm{Si}^{3}$

Apart from the substitutional fractions, the rms displacements $u_{1}\left({ }^{167 m} \mathrm{Er}\right)$ from the ideal $\mathrm{S}_{\mathrm{Ga}}$ sites were deduced as well [Figs. 2(b)-2(d)]. From our experiments it is not possible to distinguish between small static or dynamic displacements. A first observation is that, for all cases, the displacements are larger than the room temperature thermal vibration amplitude of $\mathrm{Ga}$ in $\mathrm{GaN}, u_{1}(\mathrm{Ga})=0.0739 \AA$. This means that the Er atoms are scattered around the Ga site in a broader distribution than would be expected if the main displacement to AIP license or copyright, see http://apl.aip.org/apl/copyright.jsp 
would be due to thermal vibration. Another trend is that the rms displacements decrease as the annealing temperature increases. Most likely this can be attributed to the gradual removal of crystal defects in the vicinity of the Er atoms. Such defects (e.g., $\mathrm{Ga}$ or $\mathrm{N}$ vacancies) could displace the probe atoms statically or dynamically. This assumption is supported by the observation that for all measurement axes the rms displacements in the co-implanted samples are slightly larger than those in the virgin sample. One expects that the additional implantation of $\mathrm{O}$ or $\mathrm{C}$ increases the number of crystal defects, hence also the probability that an Er atom ends up in a disordered environment. The differences are, however, only marginally larger than the experimental sensitivity. Even after $900^{\circ} \mathrm{C}$ annealing, the rms displacements still exceed the thermal vibration amplitude and a small difference between the co-implanted and virgin samples remains. This result indicates that this annealing procedure is not sufficient to remove all implantation damage. Higher temperatures and possibly high $\mathrm{N}_{2}$ pressures are needed to obtain a better recovery of the crystal. ${ }^{7,9,11}$

For each sample and annealing temperature different $u_{1}$ values are observed along the four axes, with: $u_{\perp[\overline{2} 113]}$ $>u_{\perp[0001]}>u_{\perp[\overline{1} 102]} \geqslant u_{\perp[\overline{1} 101]}$. This suggests that a preferential crystal axis for displacement of Er atoms exists. Such anisotropy might arise from the formation of defect complexes in which the Er is slightly displaced from the perfect $\mathrm{S}_{\mathrm{Ga}}$ site along a certain direction. The smallest $u_{1}$ values are found for displacements perpendicular to the [11102] and [1101] axes which are both located within the $\{11 \overline{2} 0\}$ planes, suggesting that the preferred displacement direction should lie close to these two axes. Since a similar displacement behavior is found in all samples, the influence of oxygen and carbon on erbium in $\mathrm{GaN}$ must be small. ${ }^{8}$

The nature of defects surrounding the Er atoms and their persistence after annealing could explain the observation of different $\mathrm{Er}$ photoluminescence centers ${ }^{16,17}$ even though most $\mathrm{Er}$ is located on $\mathrm{S}_{\mathrm{Ga}}$ sites. These centers are most likely specific Er-defect complexes with Er near the $\mathrm{S}_{\mathrm{Ga}}$ site, instead of erbium on different lattice sites.

To summarize, we have studied the lattice location of $\mathrm{Er}$ in $\mathrm{GaN}, \mathrm{GaN}: \mathrm{O}$, and $\mathrm{GaN}: \mathrm{C}$ using emission channeling. High fractions $(\approx 90 \%)$ of Er were found on substitutional Ga sites already after room temperature implantation. The slightly anisotropic displacements from the $S_{G a}$ sites decrease with increasing annealing temperature, but remain substantially larger than the expected thermal vibration, even after $900{ }^{\circ} \mathrm{C}$ annealing. The differences between the three samples are well within the experimental error bars, except for the rms displacements. The $\mathrm{Er}$ in the co-implanted samples thus exhibits very similar behavior as Er in virgin GaN. Our findings therefore show that co-implanting Er with $\mathrm{O}$ or $\mathrm{C}$ into $\mathrm{GaN}$ does not significantly affect the incorporation of Er into Ga sites in the case of low-dose implantation. This means that any difference in luminescence caused by co-implantation of Er with $\mathrm{O}$ or $\mathrm{C}$ into GaN cannot be attributed to a change in the lattice site location of Er, unlike in the case of $\mathrm{Er}$ in $\mathrm{Si}^{2,3}$

One of the authors (B.D.V.) acknowledges his fellowship from FWO-Vlaanderen, and U.W. from the Foundation for Science and Technology Portugal (FCT). This work was funded by the FCT Project CERN/FIS/43725/2001, and by the European Commission through the RENiBE1 (Rare Earth Doped Nitrides for High Brightness Electroluminescent Emitters) network and the HPRI Program (Large Scale Facility Contract HPRI-CT-1999-00018).

${ }^{1}$ Y. Q. Wang and A. J. Steckl, Appl. Phys. Lett. 82, 502 (2003).

${ }^{2}$ J. Michel, J. L. Benton, R. F. Ferrante, D. C. Jacobson, D. J. Eaglesham, E. A. Fitzgerald, Y. H. Xie, J. M. Poate, and L. C. Kimerling, J. Appl. Phys. 70, 2672 (1991).

${ }^{3}$ U. Wahl, A. Vantomme, J. De Wachter, R. Moons, G. Langouche, J. G. Marques, J. G. Correia, and the ISOLDE Collaboration, Phys. Rev. Lett. 79, 2069 (1997).

${ }^{4}$ U. Wahl, E. Alves, K. Lorenz, J. G. Correia, T. Monteiro, B. De Vries, A. Vantomme, and R. Vianden, Mater. Sci. Eng., B 105, 132 (2003).

${ }^{5}$ J. T. Torvik, C. H. Qiu, R. J. Feuerstein, J. I. Pankove, and F. Namavar, J. Appl. Phys. 81, 6343 (1997).

${ }^{6}$ E. Alves, M. F. da Silva, J. C. Soares, R. Vianden, J. Bartels, and A. Kozanecki, Nucl. Instrum. Methods Phys. Res. B 147, 383 (1999).

${ }^{7}$ J. M. Zavada, C. J. Ellis, J. Y. Lin, H. X. Jiang, J. T. Seo, U. Hömmerich, M. Thaik, R. G. Wilson, P. A. Grudowski, and R. D. Dupuis, Mater. Sci. Eng., B 81, 127 (2001).

${ }^{8}$ P. H. Citrin, P. A. Northrup, R. Birkhahn, and A. J. Steckl, Appl. Phys. Lett. 76, 2865 (2000).

${ }^{9}$ V. Y. Davydov, V. V. Lundin, A. N. Smirnov, N. A. Sobolev, A. S. Usikov, A. M. Emel'yanov, M. I. Makoviichuk, and E. O. Parshin, Semiconductors 33, 1 (1999).

${ }^{10}$ E. Alves, T. Monteiro, J. C. Soares, L. Santos, M. F. de Silva, J. C. Soares, W. Lojkowski, K. Kolesnikov, R. Vianden, and J. G. Correia, Mater. Sci. Eng., B 81, 132 (2001).

${ }^{11}$ T. Monteiro, J. Soares, M. R. Correia, and E. Alves, J. Appl. Phys. 89, $6183(2001)$

${ }^{12}$ M. Overberg, C. R. Abernathy, J. D. MacKenzie, S. J. Pearton, R. G. Wilson, and J. M. Zavada, Mater. Sci. Eng., B 81, 121 (2001).

${ }^{13}$ M. Dalmer, M. Restle, A. Stötzler, U. Vetter, H. Hofsäss, M. D. Bremser, C. Ronning, R. F. Davis, and the ISOLDE Collaboration, Mater. Res. Soc. Symp. Proc. 482, 1021 (1998).

${ }^{14}$ H. Hofsäss and G. Lindner, Phys. Rep. 201, 121 (1991).

${ }^{15}$ U. Wahl, Hyperfine Interact. 129, 349 (2000).

${ }^{16}$ S. Kim, S. J. Rhee, D. A. Turnbull, X. Li, J. J. Coleman, S. G. Bishop, and P. B. Klein, Appl. Phys. Lett. 71, 2662 (1997).

${ }^{17}$ H. Przybylinska, W. Jantsch, and A. Kozanecki, Mater. Sci. Eng., B 81, 147 (2001). 